\title{
Dopant atom distribution and spatial confinement of conduction electrons in Sb-doped $\mathrm{SnO}_{2}$ nanoparticles
}

\author{
C. McGinley* \\ HASYLAB/DESY, Notkestrasse 85, D-22603 Hamburg, Germany \\ H. Borchert and M. Pflughoefft \\ Institut für Physikalische Chemie, Universität Hamburg, D-22761 Hamburg, Germany \\ S. Al Moussalami \\ HASYLAB/DESY, Notkestrasse 85, D-22603 Hamburg, Germany \\ A. R. B. de Castro \\ Laboratorio National de Luz. Sincrotron, Campinas 13081-90, Brazil \\ M. Haase and H. Weller \\ Institut für Physikalische Chemie, Universität Hamburg, D-22761 Hamburg, Germany \\ T. Möller \\ HASYLAB/DESY, Notkestrasse 85, D-22603 Hamburg, Germany
}

(Received 4 July 2001; revised manuscript received 1 October 2001; published 29 November 2001)

\begin{abstract}
Colloidally prepared nanoparticles of pure and $n$-type $\mathrm{SnO}_{2}$ were deposited on $\mathrm{Au}$ foils and studied by photoelectron spectroscopy with synchrotron radiation. Substitutional $n$-type doping was achieved using $\mathrm{Sb}$ with an initial oxidation state of III or V in the chemical preparation process. The dopant concentration was $16.7 \%$. Dopant atom distributions in the nanoparticles were found by measuring the intensity ratio of the $\mathrm{Sb}$ $3 d_{3 / 2}$ to $\mathrm{Sn} 3 d_{3 / 2}$ core level photoelectron spectra as a function of the excitation energy. Sb was more evenly distributed through the nanoparticles when its initial oxidation state was $\mathrm{Sb}^{V}$ while some surface enrichment of the dopant resulted for $\mathrm{Sb}^{I I I}$ doping. High-resolution $\mathrm{Sn} 3 d$ core level spectra show a plasmon satellite feature which is due to the excitation of conduction band electrons. These exist as a free electron gas confined to the inner region of the nanoparticles.
\end{abstract}

DOI: 10.1103/PhysRevB.64.245312

PACS number(s): 79.60.Jv, 61.46.+w

\section{INTRODUCTION}

Colloidal preparation methods have been highly successful in producing semiconductor nanocrystals of precise crystal structure with size-dependent electronic and thermal properties. ${ }^{1-3}$ Surface passivation with ligand chains is essential for the stability of such II-VI and III-V nanocrystals and electronically these systems are intrinsic. To induce $n$-type conductivity in these nanocrystals by substitutional doping has not yet been achieved. A successful alternative is the method of electron injection from alkali metals at the surface $^{4}$ which results in unambiguous electron occupation in II-VI nanocrystals. Electron injection may also be used to tune the optical properties of II-VI nanocrystals so that they exhibit an electrochromic effect ${ }^{5}$ - that is, that their optical properties change in response to an electric current.

In this work we study nanoparticles which were made $n$ type by substitutional doping and also exhibit an electrochromic effect. $\mathrm{SnO}_{2}$ nanoparticles need no attached ligands as the surface of this material is structurally and electronically stable in air. Also, their surface charge is high enough to allow them to exist in a stable colloidal solution. These nanoparticles show no large band gap size dependence but a film of the nanoparticles exhibits novel electronic properties due to a strong electrochromic effect when doped $n$ type. ${ }^{6,7}$ Spe- cifically the strength of the infrared plasma absorption increases with electron injection through the particles. Related applications of $n$-type metal oxides include solar cells ${ }^{8}$ and transparent heat reflectors ${ }^{9}$ in optoelectronic devices. Nanoparticle films of this material also represent a useful system for examining the relationship between electronic and structural properties in a nanoscale system.

Nanoparticle solutions were prepared with either $\mathrm{Sb}^{I I I} \mathrm{Cl}_{3}$ or $\mathrm{Sb}^{V} \mathrm{Cl}_{5}$ as the dopant precursors in the preparation method. Two important questions relate to how dopant atoms are distributed throughout the nanoparticles and also how to quantify the concentration of conduction electrons supplied by the dopant. Dopant concentration profiles in the $60 \AA$ particles were evaluated using tunable synchrotron radiation to record core level photoelectron spectra from dopant and host atoms. By increasing the photon energy the sampling depth is increased and a simulation of the $\mathrm{Sb} 3 d_{3 / 2}$ to $\mathrm{Sn}$ $3 d_{3 / 2}$ core level intensity ratio variation with $h \nu$ yields the dopant concentration profile. This method is similar to that previously used in determining thin-film coverage in overlayer growth studies. ${ }^{10}$

Plasmon satellites were identified in the photoelectron spectra of the core levels. In measuring the relative intensity of the plasmon feature as a function of sampling depth we see that the conduction electrons are spatially confined to the 
center of the nanoparticles. From the plasmon energy an estimate of the concentration of conduction electrons in the particles was also found.

Another area of interest for heavily doped semiconductors is the problem of band gap shrinkage. Although this is a large effect for bulk metal oxide semiconductors, ${ }^{11,12}$ we find that in the surface region of the nanoparticle films this effect is negligible.

\section{EXPERIMENT}

Colloidal nanoparticles were prepared in solution by a method previously described. ${ }^{13}$ Transmission electron microscopy (TEM) measurements showed that the nanoparticles had a narrow size distribution with an average diameter of $60 \AA$. The samples are highly monodisperse with a size variation of less than $10 \%$ as estimated from the TEM images. Dopant concentrations were measured with an error of less than $1 \%$ using atomic emission spectroscopy (AES). Au foils were dip coated in the solutions and the resulting nanoparticle films were transported in air and introduced into a UHV chamber using a fast-entry load lock. Previous work has shown that the samples are not degraded on exposure to air and that the surface termination layer contains $\mathrm{OH}^{-}$ ions. ${ }^{14}$

Photoelectron spectra were recorded using beamline BW3 of the DORIS III storage ring at HASYLAB/DESY in Hamburg, Germany. Photons were tuned in the 500-1200 eV energy range for this work and photoelectron spectra collected with an Omicron EA125 hemispherical energy analyser (HEA). Samples were oriented at $45^{\circ}$ with respect to the synchrotron light and the photoelectron spectra recorded in normal emission mode. A combined instrumental resolution of $260 \mathrm{meV}$ was used for recording the high-resolution core level spectra. These were then fitted to the minimum number of Voigt functions following subtraction of a polynomial background. Photon energies and hence binding energy scales were calibrated by measuring the position of the Fermi level in Au. The width of these Fermi level spectra was also used to estimate the total experimental resolution. ${ }^{15}$

To find the Sb:Sn concentration ratio through the nanoparticles, wide-range low-resolution spectra were recorded at a series of photon energies. A combined experimental resolution of $2.0 \mathrm{eV}$ was sufficient for these measurements. These spectra covered an energy range including the $\mathrm{Sn} 3 d$ and $\mathrm{Sb}$ $3 d$ core levels so that at each photon energy the light intensity could be assumed constant for both core levels.

\section{SIMULATION METHOD}

In photoemission the intensity of a core level signal is given by

$$
I(h \nu)=c \sigma_{n l}(h \nu) S_{d e t}\left(E_{k i n}\right) \int_{V} d V \rho_{a t}(\mathbf{r}) e^{-d(\mathbf{r}) / \lambda\left(E_{k i n}\right)}
$$

where $c$ is a constant and $\sigma_{n l}$ is the atomic photoionisation cross section for the core level $n l$ which was taken from Band et al. ${ }^{16}$ We include in $\sigma_{n l}$ a small asymmetry parameter

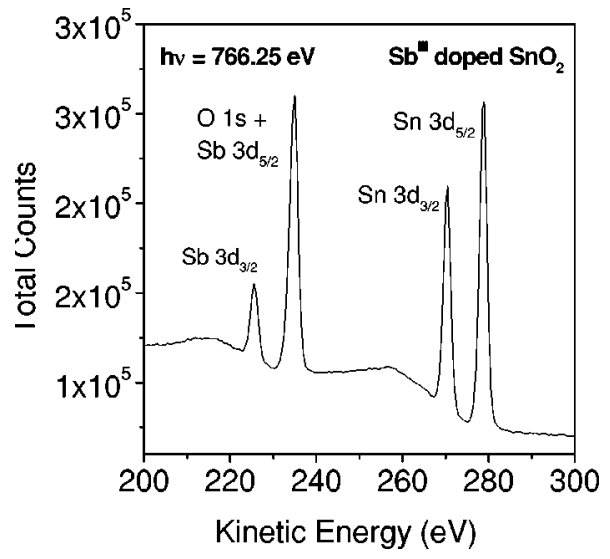

FIG. 1. Overview spectrum for $\mathrm{Sb}^{I I I}$ doped $\mathrm{SnO}_{2}$ nanoparticles showing the core level peaks used to measure the $\mathrm{Sb}$ spatial distribution.

correction, also from Ref. 16, to account for our experimental geometry. $S_{d e t}$ is the detector sensitivity function and $\rho_{a t}(\mathbf{r})$ the radial density distribution of the particular element in the nanoparticle. $d$ is the distance a primary photoelectron travels through the nanoparticle following emission at the point $\mathbf{r}$ and $\lambda\left(E_{k i n}\right)$ is the photoelectron attenuation length in $\mathrm{SnO}_{2}$. This was calculated using the expression by Tanuma et al. ${ }^{17}$ which utilizes the inelastic mean-free-path length for photoelectrons in the material.

We note that the attenuation length for photoelectrons from the $\mathrm{Sn} 3 d_{3 / 2}$ and $\mathrm{Sb} 3 d_{3 / 2}$ core levels is slightly different at any given photon energy. This is due to the $45 \mathrm{eV}$ difference in binding energy between the two levels which gives a photoelectron kinetic energy difference (see Fig. 1). Also, the sensitivity of our analyzer depends on the electron kinetic energy, the exact dependence having been studied in detail elsewhere. ${ }^{18}$ From this work we have evaluated $S_{d e t}\left(E_{k i n}\right)$ for our experimental settings.

For a wide-range photoelectron spectrum such as that shown in Fig. 1, the intensities of two core levels may be related as follows. In Eq. (1), photoemission intensties $I(h \nu)$ are divided by values for $\sigma_{n l}$ and $S_{d e t}\left(E_{k i n}\right)$ which are taken from the literature. Thus we may write an equation for the normalized photoemission intensity ratio of two core levels recorded at a given photon energy:

$$
\frac{I_{n o r m}\left(\operatorname{Sb~} 3 d_{3 / 2}\right)}{I_{n o r m}\left(\operatorname{Sn~} 3 d_{3 / 2}\right)}=\frac{\int_{V} d V \rho_{S b}(\mathbf{r}) e^{-d / \lambda\left(E_{k i n}^{S b}\right)}}{\int_{V} d V \rho_{S n}(\mathbf{r}) e^{-d / \lambda\left(E_{k i n}^{S n}\right)}}
$$

The ratio of the normalized photoemission peak intensities therefore depends only on the distribution of the atoms in the nanoparticles $\rho_{a t}(\mathbf{r})$ and the photoelectron attenuation length $\lambda\left(E_{k i n}\right)$. Since the latter varies with photoelectron kinetic energy, the observed core level intensity ratio is a function of the photon energy. This function is therefore related to the spatial distribution of the elements in the nanoparticle 
film. So by recording spectra at different energies the data may be simulated to find the dopant atom distribution in the nanoparticles.

The nanoparticles were spherical with a radius $R$ $=30 \AA$ and a narrow-size distribution. The degree of doping $D$, a function of radial postition $\mathbf{r}$, is given by

$$
D(\mathbf{r})=\frac{N_{S b}}{N_{S b}+N_{S n}} .
$$

Assuming that $D(\mathbf{r})$ shows spherical symmetry and since we know the total doping concentration $D_{\text {tot }}$, we have

$$
\int_{V} d V D(\mathbf{r})=4 \pi \int_{0}^{R} r^{2} d r D(r)=V D_{t o t} .
$$

To simulate the recorded data we divide the spherical particles into $m$ subshells whose doping concentration $D_{m}$ is systematically varied with the constraint that the total doping concentration $D_{t o t}$ remain constant. For each combination of $D_{1}, \ldots, D_{N}$ that satisfies Eq. (4) the theoretical intensity ratio of $\mathrm{Sb}$ to $\mathrm{Sn}$ is given by

$$
\frac{I_{n o r m}\left(\mathrm{Sb} 3 d_{3 / 2}\right)}{I_{n o r m}\left(\operatorname{Sn~} 3 d_{3 / 2}\right)}=\frac{\int_{V} d V D(r) e^{-d / \lambda\left(E_{k i n}^{S b}\right)}}{\int_{V} d V[1-D(r)] e^{-d / \lambda\left(E_{k i n}^{S n}\right)}} .
$$

In evaluating Eq. (5) for each photon energy at which data were recorded with the function $D(r)$ as the variable, the sum of quadratic deviations between the theoretical curve and experimental data was calculated. Finding the minimum leads to the distribution of $\mathrm{Sb}$ in the $\mathrm{SnO}_{2}$ nanoparticles.

\section{RESULTS AND DISCUSSION}

\section{A. Distribution of dopant atoms}

Figure 1 shows an overview photoelectron spectrum recorded with a resolution of $2.0 \mathrm{eV}$ and using a photon energy of $766.25 \mathrm{eV}$. The kinetic energy range covers the core level peaks of interest for estimating the dopant atom distribution in the nanoparticles. As the $\mathrm{Sb} 3 d_{5 / 2}$ peak overlaps with the O $1 s$ peak, we compare areas under the $j=3 / 2$ peaks of the $\mathrm{Sn} 3 d$ and $\mathrm{Sb} 3 d$ doublets. A polynomial background was subtracted from the core level spectra, areas integrated numerically, and each area was then normalized for $\sigma_{n l}$ and $S_{d e t}$ as described in Sec. III.

$\mathrm{Sb} 3 d_{3 / 2}: \mathrm{Sn} 3 d_{3 / 2}$ photoemsission intensity ratios are shown as a function of photon energy in Fig. 2 for $\mathrm{SnO}_{2}$ nanoparticles doped with $16.7 \% \mathrm{Sb}^{I I I}$ and $\mathrm{Sb}^{V}$, respectively. We can see immediately that there is a qualitative difference in the results for the two types of samples. At low photon energies the Sb:Sn photoemission intensity ratios are higher for the $\mathrm{Sb}^{I I I}$-prepared sample in comparison to the $\mathrm{Sb}^{V}$-based sample. Also, data for $\mathrm{Sb}^{I I I}$-prepared samples show a sharply descending slope with increasing photon energy in contrast to data for the $\mathrm{Sb}^{V}$-based samples which stay almost constant within the error bars.

Also shown in Fig. 2 are curves which result from the simulation procedure described above. The simulated Sb dis-

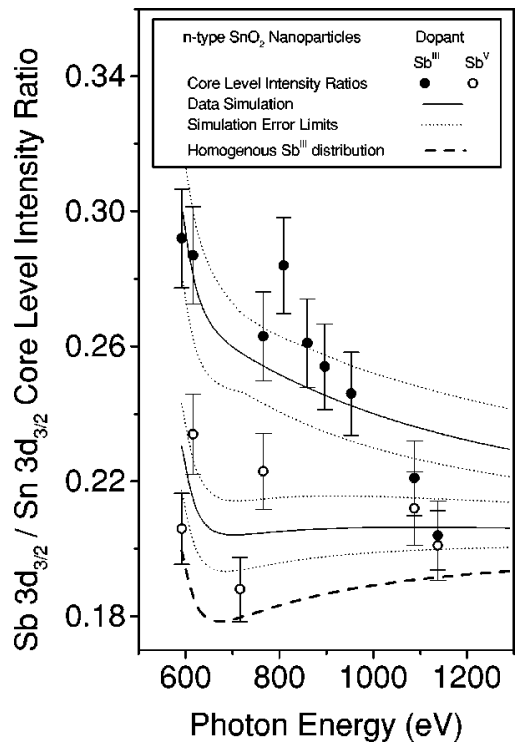

FIG. 2. Sb $3 d_{3 / 2}: \operatorname{Sn} 3 d_{3 / 2}$ core level intensity ratios as a function of photon energy for $\mathrm{SnO}_{2}$ nanoparticles doped with $\mathrm{Sb}^{I I I}$ (solid circles) or $\mathrm{Sb}^{V}$ (open circles). Simulated intensity ratios (solid line) and the error limits (dashed line) are for nanoparticles with $\mathrm{Sb}$ concentration profiles shown in Fig. 3. Also shown is the calculated intensity ratio for a homogeneous doping concentration (dashed line). This line is not horizontal as explained in the text.

tributions are shown in Figs. 3(a) and 3(b). We see in both cases that $\mathrm{Sb}$ has been successfully incorporated deep into the spherical nanoparticles. There is, however, a contrast between the two preparation methods. Sb is more concentrated at the surface when $\mathrm{Sb}^{I I I}$ is used as the dopant precursor, but for an even dopant concentration profile, synthesis with $\mathrm{Sb}^{V}$ is more successful.

In order to estimate how sensitive the fitting procedure is to changes in the $\mathrm{Sb}$ distribution, we have plotted the fits with error bars around the fitting minimum. Every distribution that leads to deviations less than 1.4 times the minimum deviation passes through the plotted error bars. From this error estimation, one can see (from Fig. 3) that the fits are less reliable in the inner part of the nanocrystal. There are two reasons for this. First, the photoelectrons emitted in the inner subshells have to pass a distance of approximately twice the mean-free-path length inside the particles. So the corresponding contribution to the peak intensity is attenuated by nearly $90 \%$. Furthermore, only $12.5 \%$ of the total number of atoms are situated in the "inner half" $(0<r<15 \AA)$ of the nanoparticles. Therefore small changes in the Sb distribution in the inner subshells do not lead to significant changes in the simulated intensity ratios. Fitting results are more reliable for the outer subshells of the nanoparticles. This region is a shell with inner and outer radii of $15 \AA$ and $30 \AA$, respectively, and contains $87.5 \%$ of the nanoparticle volume. One can clearly see the basic trends in the system. We emphasize that we have found that $\mathrm{Sb}$ is more evenly distributed in the outer region when $\mathrm{Sb}^{V}$ as opposed to $\mathrm{Sb}^{I I I}$ is used in the preparation method. There is a tendency toward surface enrichment of the dopant in the lower initial oxidation state. 

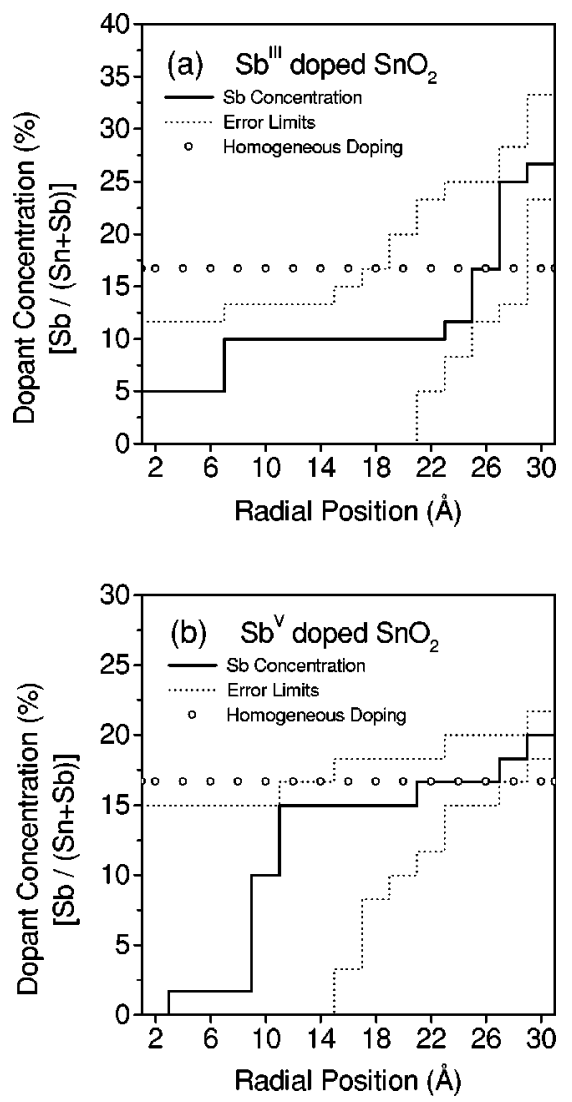

FIG. 3. Dopant atom concentration profiles in the $\mathrm{SnO}_{2}$ nanoparticles which give the best fits of the $\mathrm{Sb}: \mathrm{Sn} 3 d_{3 / 2}$ core level intensity ratio data in Fig. 2. (a) is for $\mathrm{Sb}^{I I I}$-prepared nanoparticles and (b) for $\mathrm{Sb}^{V}$-prepared nanoparticles.

It is interesting to show the expected $\mathrm{Sb}: \mathrm{Sn}$ photoemission intensity curve if we had a completely homogenous Sb distribution in the nanoparticles. This was calculated and is plotted as a dashed line in Fig. 2. The line is not horizontal. We expect this because of the binding energy difference between the two core levels and the manner in which this relates to the relative attenuation lengths of photoelectrons at different photon energies. In the energy range well above the minimum of the mean-free-path length-i.e., above $700 \mathrm{eV}$ photon energy - the sampling depth is slightly higher for Sn, the difference becoming more important at low energies. For this reason the intensity ratio corresponding to homogenous doping is slightly decreasing in this range when the energy decreases. When the energy is lowered to the vicinity of the mean free path's minimum, the difference in sampling depth becomes smaller, so that the ratio actually begins to increase. At energies below the minimum of the mean-free-path length - i.e., below $600 \mathrm{eV}$ photon energy - the ratio would even increase above the limit value at the high-energy end, because the sampling depth is then higher for $\mathrm{Sb}$.

There is of course a size variation in these nanoparticle samples which was estimated as smaller than $10 \%$ of the average diameter. To show how this may effect the simulation results we show in Fig. 4 the calculated Sb distributions if we use particle sizes $10 \%$ larger and smaller than the average value in the simulation procedure. We see that the re-
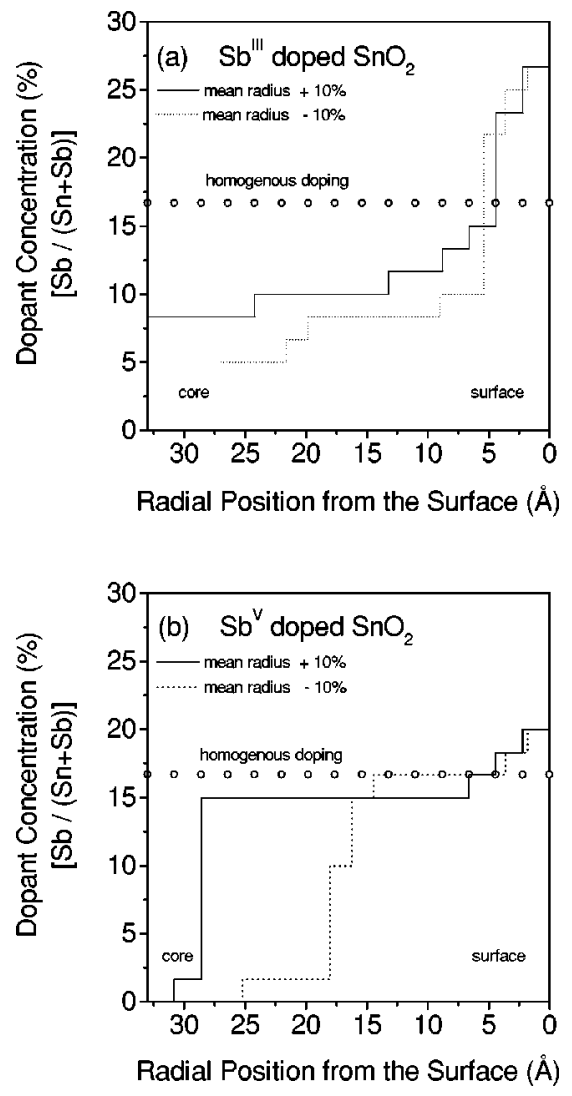

FIG. 4. Sb concentration profiles calculated by simulating the data of Fig. 2 but for different average nanoparticle sizes. Results are similar to those of Fig. 3. The size variation between sample types and the size distribution of the nanoparticles does not therefore account for the contrasting $\mathrm{Sb}$ concentration profiles in Figs. $3(\mathrm{a})$ and (b).

sulting $\mathrm{Sb}$ distributions are almost identical to those of Fig. 3. These calculations show that we can expect our results to be virtually unchanged due to particle size variation. The general result for the differences between $\mathrm{Sb}^{I I I}$ - and $\mathrm{Sb}^{V}$-prepared samples would also not be effected by a difference of up to $10 \%$ in the average particle size between the two sample types.

A further concern as to the accuracy of the simulation procedure is related to the accuracy in the dopant concentration estimation. Atomic emission spectroscopy was used to measure this and we know that the uncertainty is less than $1 \%$ for both samples. We have fitted the data of Fig. 2 assuming 1\% higher and lower Sb concentrations. Resulting Sb distributions (not shown) show that the differences between the sample types remains the same as we have shown in Fig. 3: $\mathrm{Sb}^{I I I}$-prepared samples have surface enrichment of $\mathrm{Sb}$ while $\mathrm{Sb}^{V}$ preparation gives a more homogeneous dopant distribution.

Concerning the sensitivity of this technique we should note that for the maximum kinetic energies detected the photoelectron attenuation length is some 4 times smaller than the particle diameter. In this situation $98 \%$ of the photoemission signal originates from nanoparticles at the very top of the sample, so even if the sample is deposited in three- 


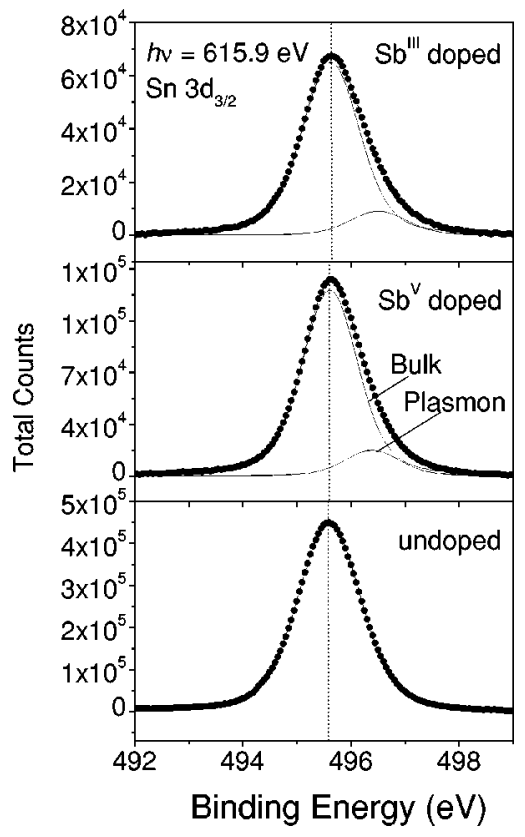

FIG. 5. Sn $3 d_{3 / 2}$ core level spectra for pure $\mathrm{SnO}_{2}$ and $\mathrm{Sb}^{I I I}$ - and $\mathrm{Sb}^{V}$-doped nanoparticles. These were recorded using a photon energy of $615.9 \mathrm{eV}$. Voigt components are associated with Sn atoms in the bulk or with a plasmon satellite. Experimental data are shown as dots only with Voigt components and their sum as a solid line.

dimensional islands, the recorded data are almost entirely dependent on the topmost layer of nanoparticles only.

\section{B. Sn $3 d_{3 / 2}$ core level spectra}

Photoemission spectra of the $\mathrm{Sn} 3 d_{3 / 2}$ core level are shown in Fig. 5 for $60 \AA \mathrm{SnO}_{2}$ nanoparticles which were doped with $\mathrm{Sb}^{I I I}$ or $\mathrm{Sb}^{V}$ as above and also for undoped nanoparticles of the same size. These spectra were recorded at a photon energy of $615.9 \mathrm{eV}$. For the pure $\mathrm{SnO}_{2}$ sample the $\mathrm{Sn}$ $3 d_{3 / 2}$ core level spectrum is fitted with one Voigt component only. Experimental data are shown as dots with the fitted Voigt function shown as a solid line. Spectra for the Sbdoped samples, being slightly asymmetric, require a second Voigt component to obtain a good fit. This fit is also shown as a solid line and reproduces the data well. A Gaussian width of $1.00 \pm 0.05 \mathrm{eV}$ was needed to reproduce the spectra in Fig. 5. This is a high value considering that the experimental broadening was only $0.26 \mathrm{eV}$ at this photon energy. We attribute this broadening to bond length variation and some structural disorder in the nanocrystals. This is typical for nanoparticles in that Winkler et al. fitted core level photoemission spectra of CdS nanocrystals with Gaussian widths of $0.6-0.7 \mathrm{eV}$ when the experimental resolution was 0.1 eV. ${ }^{19}$ The Lorentzian width originating from lifetime broadening is $0.40 \mathrm{eV}$ for all peaks in Fig. 5 .

Previously we found that in pure $\mathrm{SnO}_{2}$ nanocrystals, $\mathrm{Sn}$ atoms exist in the bulk only and that the surfaces are terminated with a layer of $\mathrm{OH}^{-}$molecules. ${ }^{14}$ For the Sb-doped nanoparticles described here there is also no evidence from the Sn $3 d$ core level spectra for a distinct surface site for $\mathrm{Sn}$ atoms. The main peak, labeled "Bulk," in these spectra is associated with $\mathrm{Sn}^{I V}$ atoms in the bulk of the nanoparticles which are typically bonded to six oxygen atoms in $\mathrm{SnO}_{2}$.

From the point of view of binding energy the main component in the $\mathrm{Sn} 3 d$ core level is, to within less than $0.10 \mathrm{eV}$, the same for all three of the nanoparticle types studied. Vertical lines in Fig. 5 illustrate this. The measured $\mathrm{Sn} 3 d_{5 / 2}$ binding energies (not shown) are higher than that found for the equivalent core level spectral peak in 3\% Sb-doped bulk $\mathrm{SnO}_{2}$ (Ref. 12) by $0.16-0.23 \mathrm{eV}$. Three causes are considered. The first is that the higher-core-level binding energy is caused by a high density of conduction electrons. This was shown by Dou et al. ${ }^{11}$ for an $n$-type $\mathrm{CdO}$ thin film where core level binding energy shifts matched the valence band shift towards the Fermi level and both were due to a band gap shrinkage typical for highly doped bulk semiconductors. ${ }^{11}$ We dismiss this below on considering the valence band spectra. The second is a more general cause for core level binding energy shifts in semiconductors. These are due to charge transfer and electrostatic interaction (Madelung) energy differences between the nanoparticle and thinfilm material. ${ }^{20}$ Metal-oxide semiconductors show a large bond length contraction at surfaces. In a simple picture, this will increase the valence charge transfer from the metal ions towards oxygen. With less valence charge for a given nuclear charge, metal atom core electrons will therefore have a higher binding energy. We expect a high degree of crystalline defects in the $\mathrm{SnO}_{2}$ nanoparticles, ${ }^{21}$ which broadens the core level spectra as described above. As bond length contraction is expected at and near these defects the metal core level features should be shifted to relatively higher binding energy for the nanoparticles.

A third reason for the shift to higher binding energy for the core level spectra might be that the core hole remaining in the nanoparticle does not relax within the photoemission time scale and so causes photoelectrons to be emitted at a kinetic energy lower than in the case of bulk material. Such a final-state effect is quite general for metal clusters on insulating surfaces ${ }^{22,23}$ as well as for free clusters. ${ }^{24}$ Here we have highly doped semiconductor nanoparticles on a metal substrate, but we cannot confirm whether or not this finalstate effect is present or not from core level spectra alone. This is discussed further below in relation to valence band spectra.

The peak on the high-binding-energy side of the spectra in Fig. 5 is due to a loss process caused by excitation of conduction band electrons. Photoemission plasmon satellites were observed for $3 \% \mathrm{Sb}$-doped $\mathrm{SnO}_{2}$ thin films by Egdell et $a l .{ }^{12}$ They interpreted the two core level peaks as being due to a competition between initial- and final-state effects in photoemission. It is the conduction electron gas which may screen the core hole in the crystal lattice so that an intrinsic conduction band plasmon is not created. Competition between the creation and noncreation of plasmons was a striking effect in the core level spectroscopy for the doped material. The plasmon satellite was predominantly due to an intrinsic process and the $\mathrm{Sn} 3 d$ satellite peaks had an integrated area of 3 times that of the main peak for a photon energy of $1486 \mathrm{eV}$. Figure 6 shows the plasmon/bulk Voigt intensity ratio as a function of photon energy. Extrapolating 


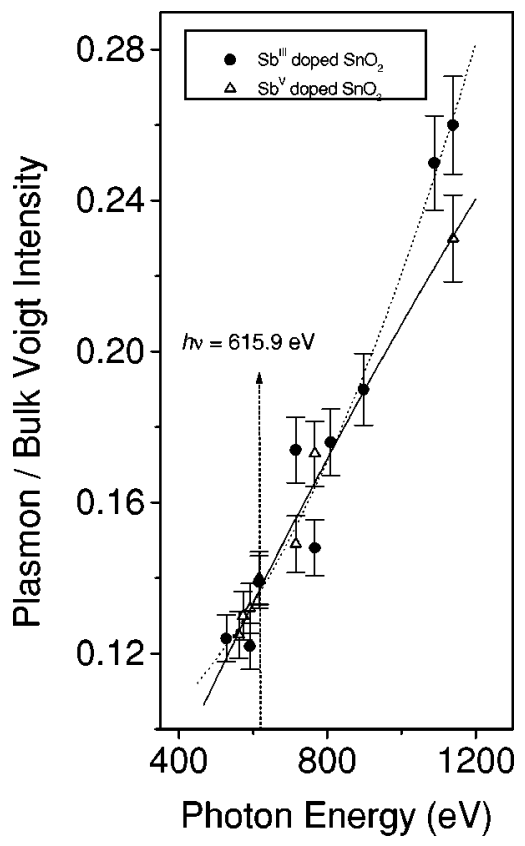

FIG. 6. Sn $3 d$ Voigt intensity ratios (plasmon/bulk) for the Sbdoped nanoparticles as a function of photon energy. A vertical dashed line marks the photon energy used to record the spectra of Fig. 5.

Fig. 6 to the same photon energy used by Egdell et al., ${ }^{12}$ our plasmon/bulk intensity ratio is a factor of 10 less than theirs. The apparent disparity is explained by the fact that in the nanocrystalline system conduction electrons are confined to the central region of the nanoparticles. This was shown for nanocrystalline layers of $\mathrm{SnO}_{2}: \mathrm{Sb}$ in an aqueous electrolyte $^{7,25}$ where band bending towards the semiconductor's surface created a well for the conduction electrons, therefore confining them to the central region of the nanoparticle. Here we have our samples in the vacuum chamber but the result from the point of view of confinement is the same. As conduction electrons are confined to the center of the particles, the contribution of the plasmon satellite to the core level spectra depends on the bulk sensitivity of our measurements. At the highest photon energy used to record spectra, photoelectrons from the Sn $3 d$ core level would have a kinetic energy of $\sim 650 \mathrm{eV}$ and so have an attenuation length of $\sim 13 \AA$ in the material. ${ }^{17}$ Photoelectrons originating from deeper in the nanoparticles will also contribute to the primary $\mathrm{Sn} 3 d$ photopeak but electrons originating from the surface should always dominate. Figure 6 therefore demonstrates the confined nature of the conduction electrons in the nanoparticles.

The $\mathrm{Sb} 3 d_{3 / 2}$ core level spectra, published separately, ${ }^{14}$ are similar to the $\operatorname{Sn} 3 d_{3 / 2}$ data shown here in that they have one principal component with a satellite peak showing the same kinetic energy shift and the same photon energy dependence (within error) as that of Fig. 6. Sb has therefore one chemical environment uniquely observed by photoemission and hence no chemical or surface shifts caused by alloying or reduced coordination number.

For both $\mathrm{Sn} 3 d$ and $\mathrm{Sb} 3 d$ core levels therefore we see no surface core level shifts. The photon energy dependence of the low-kinetic-energy peak is such that if it were associated with a chemical shift the atoms involved would be deep in the interior of the nanoparticles and with an alloying effect relying on $\mathrm{Sn}$ bonding to fewer $\mathrm{O}$ atoms in the interior and a similar situation for $\mathrm{Sb}$. This is impossible as a reduced $\mathrm{O}$ coordination in one region for $\mathrm{Sn}$ should have the opposite effect for $\mathrm{Sb}$ atoms. That the second component is shifted to low kinetic energy, for both $\mathrm{Sn}$ and $\mathrm{Sb}$, allied to the photon energy dependence eliminates the possibility of interpreting the spectra in terms of chemical or surface shifts. The only surface chemical shift in the system is for the $\mathrm{O} 1 \mathrm{~s}$ level and is caused by a surface termination layer of $\mathrm{OH}^{-} .14$

We associate the plasmon loss peak in photoemission with the creation of a bulk plasmon. In general, for semiconductor nanoparticles, surface plasmons are observed only at a very low intensity for Si clusters ${ }^{26}$ and not at all for CdS quantum dots. ${ }^{27}$ Although these authors used electron energy loss spectroscopy (EELS) to study valence band plasmons which have a particle-size-dependent excitation energy in the 20-40 eV energy range, the same absence of a surface plasmon is expected here. No improvement in the fitting procedure results from including a third Voigt function to represent a surface plasmon peak.

Figure 6 shows that as we increase bulk sensitivity in photoemission, the plasmon feature increases in intensity. Some such increase is expected for plasmons produced in an extrinsic process but the photoemission process also produces intrinsic plasmons. The latter are not expected to show an excitation energy dependence and will dominate the loss process in a material with a relatively low density of conduction electrons such as a degenerately doped semiconductor. This dominance of the intrinsic process justifies our conclusion that the conduction electrons are confined deep inside the nanoparticles. We avoid fitting the data in Fig. 6 as the required curve would be a convolution of a depth sensitivity curve for photoemission and a curve representing the photon energy dependence of the extrinsic plasmon contribution. The latter relies on parameters which for this material we are unsure, ${ }^{28}$ so we turn our attention to the actual carrier concentration.

In our data the plasmon satellite peak are shifted from the Sn $3 d$ primary peak by $0.87 \pm 0.05 \mathrm{eV}$ and $0.81 \pm 0.05 \mathrm{eV}$ for the samples prepared with $\mathrm{Sb}^{I I I}$ and $\mathrm{Sb}^{V}$, respectively. As this peak is predominantly due to bulk plasmon production, this energy shift is equal to a plasmon energy $\hbar \omega_{p}$ where

$$
\omega_{p}^{2}=\frac{N e^{2}}{m^{*} \epsilon_{0} \epsilon_{\infty}} .
$$

We use a value for the high-frequency dielectric constant $\epsilon_{\infty}$ of $3.9,{ }^{29}$ which is a value averaged over the primary cystallographic directions. $m^{*}$ is the effective mass for conduction band electrons and $N$ their concentration. These however are interdependent; i.e., $m^{*}$ increases linearly with $N$ :

$$
m^{*}=m_{0}^{*}+c N
$$

This was shown by Sanon et al. ${ }^{30}$ in collating optical absorbtion data for highly doped $\mathrm{SnO}_{2}$ from many groups and 
we use their results here. $m_{0}^{*}$ is the effective mass for nondoped $\mathrm{SnO}_{2}$ and $c$ is the slope of a linear fit. Values for these were extracted from Ref. 30. Combining Eqs. (6) and (7) and rewriting for $N$ explicitly we have

$$
N=\frac{\omega_{p}^{2} \epsilon_{0} \epsilon_{\infty} m^{*}}{e^{2}}=A\left(m_{0}^{*}+c N\right)
$$

or

$$
N=\frac{A m_{0}^{*}}{1-A c}
$$

This gives values for $N$ of $(6.80 \pm 0.80) \times 10^{20} \mathrm{~cm}^{-3}$ and $(5.64 \pm 0.70) \times 10^{20} \mathrm{~cm}^{-3}$ for $\mathrm{Sb}^{I I I}$ and $\mathrm{Sb}^{V}$ dopant precursors, respectively. As other groups have found, not all $\mathrm{Sb}$ atoms donate an electron to the conduction band in degenerately doped $\mathrm{SnO}_{2}{ }^{12,13,31}$ This is because a large amount of $\mathrm{Sb}$ exists as an $\mathrm{Sb}^{I I I}$-like lone pair state which traps electrons. ${ }^{31}$ We find here that the size of this effect is almost independent of the chemical form of Sb used in the nanoparticle synthesis. The ratio of conduction electrons to dopant atoms is approximately 0.16 and 0.12 for $\mathrm{Sb}^{I I I}$ - and $\mathrm{Sb}^{V}$-prepared samples, respectively. The uncertainty in this ratio is due to imprecise knowledge of the exact volume density of the nanoparticles since some bond length variation is expected as mentioned above. Although this relates to crystal defects, the average bond length will also be affected by the $\mathrm{Sb}$ doping especially since $\mathrm{Sb}^{I I}$-like ions are expected to reside at interface defects.

\section{Valence band spectra}

Figure 7 shows valence band spectra from films of pure gold (a) and nanoparticles of pure $\mathrm{SnO}_{2}$ on gold (b) and $\mathrm{Sb}^{I I I}$-doped $\mathrm{SnO}_{2}$ nanoparticles on gold (c). The gold valence band spectrum shows emission up to the Fermi level $\left(E_{f}\right)$. No gold valence features are seen in the pure $\mathrm{SnO}_{2}$ nanoparticle spectrum as the layer was thick enough to scatter all photoelectrons from the gold. By extrapolating the leading edge of the valence band in the spectrum 7(b) we see that the band edge is $3.60 \pm 0.05 \mathrm{eV}$ from the Fermi level. This corresponds to the bulk semiconductor band gap for pure cassiterite $\mathrm{SnO}_{2}$. Therefore the Fermi level energy coincides with that of the conduction band minimum, implying that even the pure $\mathrm{SnO}_{2}$ nanoparticles are $n$ type. This is due to defect vacancies at oxygen lattice sites which shifts the Fermi level to the bottom of the conduction band. ${ }^{12}$

The pure $\mathrm{SnO}_{2}$ valence band spectrum also shows an appreciable "tail off" into the band gap which we associate with a surface state related to the $\mathrm{OH}^{-}$groups which terminate the surface. ${ }^{14}$ This density of states in the band gap is not due to the band gap variation caused by some small nanoparticle size distribution since the band gap of $\mathrm{SnO}_{2}$ does not vary with nanoparticle size. This is a consequence of the Wannier exciton in the material having an almost zero oscillator strength.

Spectrum 7(c) is taken from a thin nanoparticle layer of intentionally doped $\mathrm{SnO}_{2}$. The spectrum shows valence band

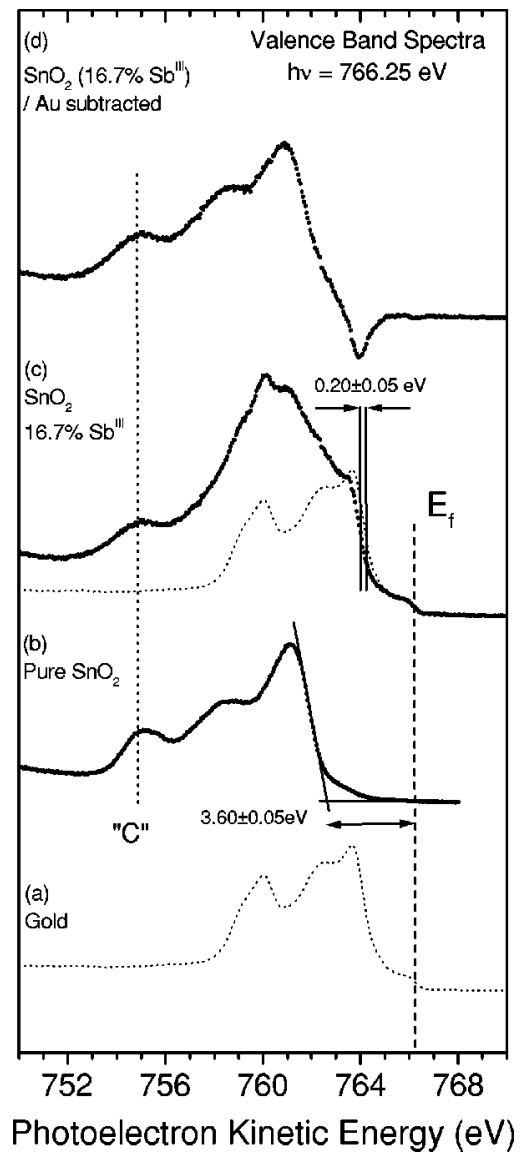

FIG. 7. Valence band spectra for pure $\mathrm{Au}$ and pure and $\mathrm{Sb}^{I I I}$-doped $\mathrm{SnO}_{2}$ nanoparticles: (a), (b), and (c) respectively. (d) is the spectrum (c) with the normalized Au spectrum (a) subtracted. For (d) the "negative intensity" above the valence band maximum is due a shift in the valence band maximum of $\mathrm{Au}$ when the $\mathrm{SnO}_{2}$ nanoparticles were deposited as explained in the text.

features from both gold and $\mathrm{SnO}_{2}$ and the gold valence band is repeated for comparison after normalization to the height of the emission at $E_{f}$. A slight offset between the band edges is obvious. This is because for the metal/semiconductor interface conduction electrons from the metal lower their energy in becoming trapped at the interface in semiconductor acceptor states such as $\mathrm{Sb}^{I I I}$ atoms. Valence electrons in the gold will therefore have a slightly higher binding energy than in the case of a clean gold surface. The shift effects photoemission from the $A u$ substrate only and is $0.20 \pm 0.05 \mathrm{eV}$.

It is interesting to discuss this slight binding energy shift in view of final-state effects observed for metal clusters on an amorphous carbon surface. This was considered above in discussing the binding energies of the $\operatorname{Sn} 3 d_{3 / 2}$ core levels. Wertheim et al. showed that as the cluster size decreases, a progressively larger influence of the core hole produced in photoemission is exerted on the outgoing electron. ${ }^{22}$ All photoemission features are shifted to higher binding energies for smaller clusters. In our case we see that it is the photoemission for the substrate Au itself which is shifted for the reason given above and so we are inclined to rule out the possibility of this final-state effect for highly doped semicondcutor nanoparticles in interpreting our results. This could of course 
be clarified by investigating a series of samples with progressively smaller nanoparticle sizes.

It is this shift in the leading edge of the Au valence band which causes difficulty if the two spectra in Fig. 7(b) are subtracted as is shown in Fig. 7(d). We cannot find the position of the valence band maximum from this "gold subtracted" data but we can compare the energy position of the feature labeled $C$ between the different spectra. Peak $C$ is derived from states based on $\mathrm{Sn} 5 s-\mathrm{O} 2 p$ bonding orbitals. ${ }^{32}$ This feature is unaffected by emission from gold in all spectra. Between the pure and $\mathrm{Sb}$-doped $\mathrm{SnO}_{2}$ samples there is no spectral energy shift in the feature $C$ with respect to the Fermi level. This is unusual as we would expect degenerately doped semiconductors to show band gap widening due to the Burstein-Moss shift with a further band gap reduction due to many-body effects such as electron-electron and electron-impurity interactions. ${ }^{30}$ These effects combine so as to reduce the band gap from that of undoped material. Using photoemission, Dou et al. demonstrated this for $n$-type thin-film CdO. ${ }^{11}$ Band gap renormalization or shrinkage was observed. This should apply to other highly doped posttransition-metal oxide semiconductors such as $\mathrm{SnO}_{2}$ as shown in Ref. 12. Band gap shrinkage was observed despite a shift in the valence band to a higher binding energy because the width of the occupied part of the conduction band is larger than this shift.

Our results suggest that for nanoparticles, band gap narrowing due to a high level of $n$-type doping is negligible in that part of the sample which contributes most photoelectrons to the valence band spectrum. Photoelectrons originating from near the surface contribute most to the valence band spectrum of Fig. 6(c). With no measured band gap shrinkage we infer that there are effectively no conduction band electrons in the surface region of the nanoparticles. This concurs with the Sn $3 d_{3 / 2}$ core level photoemission results described above. Conduction electrons are confined and so their effect in narrowing the band gap, if any, is deep in the nanoparticles but not at the surface.

\section{CONCLUSION}

We have measured the spatial distribution of Sb dopant atoms in n-type $\mathrm{SnO}_{2}$ nanoparticles. For doping with both $\mathrm{Sb}^{I I I}$ and $\mathrm{Sb}^{V}$, dopant atoms are successfully incorporated through the nanoparticles with a strong surface enrichment in the case of $\mathrm{Sb}^{I I I}$ doping. The carrier concentrations are $(6.80 \pm 0.80) \times 10^{20} \mathrm{~cm}^{-3}$ and $(5.64 \pm 0.70) \times 10^{20} \mathrm{~cm}^{-3}$ for $S b^{I I I}$ and $S b^{V}$ dopant precursors, respectively. Conduction electrons are confined to the central region despite the fact that the dopant atoms are incorporated throughout the nanoparticles. Even in the case of $\mathrm{Sb}^{I I I}$ doping where the dopant atoms are slightly more concentrated at the surface, conduction electrons are confined to the central region. We also find that band gap narrowing due to degenerate doping is not present near the nanoparticle surface.

\section{ACKNOWLEDGMENTS}

We would like to thank A. A. Cafolla of Dublin City University for providing the photoemission curve fitting software TCFIT and J. Westermann of Omicron Vakuumphysik for technical advice.
*Corresponding author. Electronic mail: mcginley@mail.desy.de

${ }^{1}$ H. Weller, Adv. Mater. 5, 88 (1993).

${ }^{2}$ A. P. Alivisatos, Science 271, 933 (1996).

${ }^{3}$ L. Brus, in Nanotechnology, edited by G. L. Timp (AIP, Melville, NY, 1999).

${ }^{4}$ M. Shim and P. Guyot-Sionnest, Nature (London) 407, 981 (2000).

${ }^{5}$ C. Wang, M. Shim, and P. Guyot-Sionnest, Science 291, 2390 (2001).

${ }^{6}$ U. zum Felde, M. Haase, and H. Weller, J. Phys. Chem. B 104, 9388 (2000).

${ }^{7}$ G. Boschloo and D. Fitzmaurice, J. Phys. Chem. B 103, 3093 (1999).

${ }^{8}$ B. O'Regan and M. Grätzel, Nature (London) 353, 737 (1991).

${ }^{9}$ G. Frank, E. Kauer, and H. Kvstlin, Thin Solid Films 77, 107 (1981)

${ }^{10}$ L. C. Feldman and J. W. Mayer, Fundamentals of Surface and Thin Film Analysis (Elsevier, New York, 1986).

${ }^{11}$ Y. Dou, T. Fishlock, R. G. Egdell, D. S. L. Law, and G. Beamson, Phys. Rev. B 55, R13 381 (1997).

${ }^{12}$ R. G. Egdell, J. Rebane, T. J. Walker, and D. S. L. Law, Phys. Rev. B 59, 1792 (1999).

${ }^{13}$ J. Rockenberger, U. zum Felde, M. Tischer, L. Tröger, M. Haase, and H. Weller, J. Chem. Phys. 112, 4296 (2000).

${ }^{14}$ C. McGinley, S. Al Moussalami, M. Riedler, M. Pflughoefft, H. Borchert, M. Haase, A. R. B. de Castro, H. Weller and T. Möller, Eur. Phys. J. D 16, 225 (2001).
${ }^{15}$ H. Hövel, T. Becker, D. Funnemann, B. Grimm, C. Quitmann, and B. Reihl, J. Electron Spectrosc. Relat. Phenom. 88, 1015 (1998).

${ }^{16}$ I. M. Band, Yu. I. Kharitonov, and M. B. Trzhaskovskaya, At. Data Nucl. Data Tables 23, 443 (1979).

${ }^{17}$ S. Tanuma, C. J. Powell, and D. R. Penn, Surf. Interface Anal. 21, 165 (1993).

${ }^{18}$ P. Ruffieux, P. Schwaller, O. Gröning, L. Schlapbach, P. Gröning, Q. C. Herd, D. Funnemann, and J. Westermann, Rev. Sci. Instrum. 71, 3634 (2000).

${ }^{19}$ U. Winkler, D. Eich, Z. H. Chen, R. Fink, S. K. Kulkarni, and E. Umbach, Chem. Phys. Lett. 306, 95 (1999).

${ }^{20}$ W. Mönch, Semiconductor Surfaces and Interfaces (SpringerVerlag, Berlin, 1993), pp 97-100.

${ }^{21}$ X. Pan and J. G. Zheng, in Polycrystalline Thin Films-Structure, Texture, Properties and Applications III, edited by S. M. Yalisove, B. L. Adams, J. S. Im, Y. Zhu, and F. R. Chen, Mater. Res. Soc. Symp. Proc. No. 472 (Materials Research Society, Pittsburgh, 1997), p. 87.

${ }^{22}$ G. K. Wertheim, S. B. DiCenzo, and D. N. E. Buchanan, Phys. Rev. B 33, 5384 (1986).

${ }^{23}$ H. Hövel, B. Grimm, M. Pollmann, and B. Reihl, Phys. Rev. Lett. 81, 4608 (1998).

${ }^{24}$ M. Seidl, K.-H. Meiwes-Broer, and M. Brack, J. Chem. Phys. 95, 1295 (1991).

${ }^{25}$ T. Nütz and M. Haase, J. Phys. Chem. B 104, 8430 (2000). 
${ }^{26}$ M. Mitome, Y. Yamazaki, H. Takagi, and T. Nakagiri, J. Appl. Phys. 72, 812 (1992).

${ }^{27}$ P. N. H. Nakashima, T. Tsuzuki, and A. W. S. Johnson, J. Appl. Phys. 85, 1556 (1999).

${ }^{28}$ S. A. Flodstrom, R. Z. Bachrach, R. S. Bauer, J. C. McMenamin, and S. B. M. Hagström, J. Vac. Sci. Technol. 14, 303 (1977).

${ }^{29}$ Landolt Börnstein Data Collection, http://www.springer.de/.
${ }^{30}$ G. Sanon, R. Rup, and A. Mansingh, Phys. Rev. B 44, 5672 (1991).

${ }^{31}$ C. S. Rastomjee, R. G. Egdell, M. J. Lee, and T. J. Tate, Surf. Sci. Lett. 259, 769 (1991).

${ }^{32}$ J. M. Themlin, R. Sporken, J. Darville, R. Caudano, J. M. Gilles, and R. L. Johnson, Phys. Rev. B 42, 11914 (1990). 